\title{
The degree of hepatic arterial blood supply of portal vein tumor thrombus in patients with hepatocellular carcinoma and its impact on overall survival after transarterial chemoembolization
}

\author{
Juxian Sun ${ }^{1, *}$, Jie Shi ${ }^{1, *}$, Bin Huang ${ }^{2, *}$, Fantian Cheng ${ }^{1,3}$, Weixing Guo ${ }^{1}$, Wan Yee \\ Lau $^{1,4}$ and Shuqun Cheng ${ }^{1}$ \\ ${ }^{1}$ Department of Hepatic Surgery VI, Eastern Hepatobiliary Surgery Hospital, Second Military Medical University, Shanghai, China \\ ${ }^{2}$ Department of Imaging, Eastern Hepatobiliary Surgery Hospital, Second Military Medical University, Shanghai, China \\ ${ }^{3}$ Department of Hepatopancreatobiliary Surgery I, The Central Hospital of Wuhan, Tongji Medical University of Science and \\ Technology, Wuhan, China \\ ${ }^{4}$ Faculty of Medicine, The Chinese University of Hong Kong, Shatin, New Territories, Hong Kong SAR, China \\ *These authors contributed equally to this work \\ Correspondence to: Shuqun Cheng, email: chengshuqun@aliyun.com
}

Keywords: blood supply, portal vein tumor thrombus, hepatocellular carcinoma, TACE

Received: February 08, $2017 \quad$ Accepted: June 29, $2017 \quad$ Published: August 01, 2017

Copyright: Sun et al. This is an open-access article distributed under the terms of the Creative Commons Attribution License 3.0 (CC BY 3.0), which permits unrestricted use, distribution, and reproduction in any medium, provided the original author and source are credited.

\section{ABSTRACT}

Purpose: To investigate the degree of arterial blood supply of portal vein tumor thrombus (PVTT) in patients with hepatocellular carcinoma (HCC), and to evaluate its impact on overall survival after transarterial chemoembolization using lipiodol $+/-$ gelatin sponge particles (TACE).

Results: Of the 10 patients who underwent surgery, the number of patients with good/mild/poor staining of PVTT by methylene blue were 3, 4, and 3, respectively. The degrees of methylene blue staining in these patients correlated well with the degrees of accumulation of lipiodol in PVTT in these patients, i.e. good/mild/poor in 3,4 , and 3 patients, respectively. For the 77 patients who underwent TACE as treatment, they were divided into 2 groups: good accumulation of lipiodol $(n=27)$ and mild/poor accumulation of lipiodol $(n=50)$ on CT. The overall median survival between the 2 groups was 10.0 months vs 2.7 months, $(p<0.001)$. Multi-variable analysis showed degree accumulation of Lipiodol (OR, 2.057; 95\% CI,1.414-2.993; $p<0.001$ ) to be an independent prognostic factor.

Patients and Methods: Patients with HCC with PVTT who underwent surgical resection received preoperative TACE. At operation, arterial injection of methylene blue into the common hepatic artery was carried out. During the study period, other patients with unresectable HCC with PVTT were treated with TACE.

Conclusion: In about $1 / 3$ of patients with HCC with PVTT, the arterial blood supply from the hepatic artery to the PVTT was good. These patients responded better to TACE than those patients with mild/poor arterial supply.

\section{INTRODUCTION}

Hepatocellular carcinoma (HCC) is the sixth most common cancer and the third most common cause of cancer-related death worldwide [1]. Though imaging techniques have improved, HCC is commonly diagnosed at intermediate or advanced stages. Portal vein tumor thrombus (PVTT), which is the leading cause of poor prognosis of $\mathrm{HCC}$, is present in $44.0 \%$ to $62.2 \%$ of patients [2]. Controversies exist in the management of patients with HCC with PVTT [3,4]. Sorafenib is the only drug which is recommended by EASL and AASLD for patients with PVTT bu it can only improve survival for about 3 months [5]. Resectional surgery can offer a chance of a cure, but the tumor recurrence rate is high. Transarterial chemoembolization (TACE) is a useful 
palliative treatment for patients with unresectable HCC and it is based on HCCs being mainly supplied by the hepatic artery. Major PVTT in patients with HCC is an important risk factor of poor prognosis, but it is no longer considered to be an absolute contraindication to TACE. In selected patients with PVTT with good hepatic reserve and good collateral circulation around the porta hepatis, TACE can safely be carried out. The 30-day mortality is reported to be lower than $1.2 \%$ since 2010 [6]. Clinically, it is more important to control the progress of PVTT than the liver tumor because as PVTT grows the main portal vein can become blocked. Complete/partial blockage of the main portal vein results in decrease blood flow to the liver with rapid deterioration in liver function, development of severed ascites, portal hypertension with bleeding esophageal haemorrhage, and rapid intra and extrahepatic metastases. For patients with HCC with PVTT, although there was no report on complete response (CR) to TACE, partial response (PR) has been reported in $19.5 \%$ to $26.3 \%$ and stationary disease (SD) $42.5 \%$ to $62.7 \%$ [7]. The median survival between responders $(10.5 \mathrm{mo})$ and non-responders (5.5 mo) to TACE can differ significantly [8]. Previous study [9] has proved that there would be relationship between response of PVTT and survival after radioembolization. The aim of this study to find whether the degree of hepatic arterial blood supply of PVTT is related to overall survival after TACE for patients with HCC with PVTT.

\section{RESULTS}

The arterial methylene blue dyeing test was carried out on 10 patients who underwent preoperative TACE 4 weeks prior to surgical resection (Figure 1A). The average length of PVTT was $2.7 \mathrm{~cm}$. Of the 10 patients, the PVTT was dyed by methylene blue to be good in 3 patients, mild in 4 and poor in 3. The degrees of methylene blue staining in these patients correlated well with the degrees of accumulation of lipiodol in PVTT as shown on CT, i.e. good $/ \mathrm{mild} /$ poor in 3,4 , and 3 patients, respectively (Table 1). Thus, more than two thirds of patients with PVTT were dyed with methylene blue (Figure 1B). Under microscopy, methylene blue was seen in small arterioles (Figure 1C). However, only about one third of the patients had good staining of PVTT by methylene blue to suggest good blood supply of PVTT coming from hepatic artery.

77 patients with unresectable $\mathrm{HCC}$ with PVTT underwent TACE for palliation. There were 69 men and 8 women with a median age of 49.8 years (range 29-73 years). 47 patients $(61.0 \%)$ received one session of TACE, and $30(39.0 \%)$ had more than one session. The median survival was 5.9 month for all the patients. When these patients were divided into 2 groups according to the degree of accumulation of Lipiodol in the PVTT as shown on CT (type IV + type III, the low accumulation group, $n=28+$ $22=50$; and type II + type I, the high accumulation group, $n=8+19=27)$, there were no significant differences between the 2 groups in age, gender, hepatitis serology, liver function parameters, AFP level, number of TACE sessions, tumor characteristics and PVTT classification (Table 2).

The Kaplan-Meier survival curves comparing the 77 patients with PVTT showing good accumulation of Lipiodol versus mild/poor accumulation of Lipiodol as shown on CT scan are shown in Figure 2. The overall survival of patients with types III or IV PVTT (the low lipiodol accumulation group) was significantly worse than patients with types I or II (the good lipiodol accumulation group). The median survival was 3.7 month vs. 10.0 month, respectively, $P<0.001)$. About one third of patients with PVTT. i.e. the I+II patients $(n=27)$ had significantly better overall survival than the type III + IV patients $(n=50)$ after TACE. There is also a tendency that the overall survival of type I was longer than type II patients (median survival 12.5 month vs. 8.7 month, HR 2.252, $P=$ 0.089) (Figure 3). Thus, TACE produced the best survival in patients with PVTT which showed homogeneous accumulation of lipiodol on CT scan (Figure 4).

Survival of the 4 classes of lipiodol classification according to the 4 classes of PVTT are shown in Table 3.

Univariable analysis was used to evaluate any possible correlations between survival and 11 dichotomized variables for all the 77 patients (Table 4). Results show that both tumor diameter and degree of accumulation of Lipiodol are independent prognostic factors. In multivariate analysis, tumor diameter (HR, 1.106; 95\% CI,1.011-2.211; $P=0.029)$, degree of accumulation of Lipiodol (HR, 2.057; 95\% CI,1.414$2.993 ; P=0.001)$ and AST (HR, $1.107 ; 95 \%$ CI, $1.008-$ $1.027 ; P=0.002$ ) were found to be independent prognostic factors. After making a sensitivity analysis by forcing the type of PVTT into the model, we found that the type of PVTT was not an independent prognostic factor (HR, 1.426; 95\% CI,0.901-2.258; $P=0.130$ ).

\section{MATERIALS AND METHODS}

\section{Patients}

From July 2010 to January 2012, all patients with HCC with PVTT who were treated in the Department of Hepatic Surgery VI at the Eastern Hepatobiliary Surgery Hospital were included into this study, retrospectively. The extent of PVTT was classified into: type I, involvement of segmental/sectoral branches of portal vein; type II, involvement of left or right portal vein; type III, involvement of main portal vein; and type IV, involvement of superior mesenteric vein.

Patients with resectable HCC with PVTT were offered surgical resection. The inclusion criteria for surgical resection were: 1) resectable HCC; 2) Type II/ III PVTT; 3) Child-Pugh A liver function, 4) no hepatic 
vein invasion and/or extrahepatic spread. These patients were treated with preoperative TACE 4 weeks before surgery. The degree of lipiodol accumulation in PVTT was assessed as in the patients who had unresectable HCC with PVTT treated by TACE (please see latter part of the study). All patients were given detailed information on the pros and cons of surgical resection and TACE, including treatment efficacies and potential adverse effects. Written informed consent was obtained from all the patients for their data to be used for research purposes. The study was approved by the Ethics Committee of the Eastern Hepatobiliary Surgery Hospital.

\section{The surgical procedure}

Surgery was performed through a right subcostal incision with a midline extension. Intraoperative ultrasound was done as a routine to confirm the preoperative findings. After mobilization of the liver and dissection of the structures in the porta hepatis, the proper hepatic artery was isolated and and $20 \mathrm{ml}$ of methylene blue (Shanghai X-Y Biotechnology) was injected through the artery using a 23 guage needle. Pringle's maneuver was then applied to occlude the blood inflow of the liver using continuous clamping. The planned liver resection was carried out in the usual manner. All grossly detected lesions were resected. The PVTT was surgically treated according to the location and extent of PVTT. For 6 patients with a PVTT which was located within the resected region, the PVTT was resected en bloc with the tumor. For 4 patients with a PVTT which had extended beyond the resection line, the main portal vein was opened and the PVTT was extracted from the portal vein. After flushing with normal saline and confirming that no PVTT remained, the incision in the portal vein was closed with a continuous 5-0 prolene suture. The porta hepatis clamp time in all the patients was less than 30 minutes.

The resected specimens were studied by two pathologists. For each pathologist, photographs of the transverse, sagittal and coronal sections of the specimens were taken on the liver tumor and on the PVTT. These photographs were then compared with a background showing a grid with one millimeter squares. The areas which had been dyed with methylene blue were counted from the whole PVTT areas on the 3 sections. If more than 2 sections were stained more than $50 \%$ of the areas, then the arterial blood supply of PVTT was classified as good. If only one section was stained more than $50 \%$ of the areas, then the arterial blood supply of PVTT was classified as mild. Otherwise, they were classified as poor. The average of the two values taken by the 2 pathologists was used as the final value.

\section{The TACE procedure}

During the study period, patients with unresectable HCC with PVTT underwent TACE. The following patients were included into this study: 1) unresectable HCC with PVTT; 2) type II/III PVTT; 3) Child-Pugh liver function A or B; 4) no hepatic vein or inferior vena cava invasion; 4) no previous treatment for $\mathrm{HCC}$ and no other malignancies.

TACE was performed using the Seldinger technique. Celiac and superior mesenteric arteriograms were routinely performed to show the hepatic arterial and portal venous anatomy. The catheter was then inserted to the tumor-feeding branches of the hepatic artery. Selective arteriograms were performed to demonstrate the feeding artery of PVTT. In patients with an arterioportal shunt, embolization with gelatin sponge particles was performed to occlude the shunt. Mitomycin C(MMC) $20 \mathrm{mg}$, hydroxycamptothecine $10 \mathrm{mg}$ and Lipiodol $10-30 \mathrm{ml}$ (at $1-2 \mathrm{ml} / \mathrm{cm}$ of the tumor diameter) were then given. In patients with type III PVTT, care was taken not to completely block the hepatic artery.

CT scans were taken a day following TACE. The accumulation of lipiodol in the PVTT was classified as type 1, homogeneous; type II, slight defective; type III, inhomogeneous; and type IV, only slight accumulation ${ }^{10}$.

\section{Follow up}

All patients were followed-up by the same team of clinicians. Post-treatment follow-up including ultrasonography, serum biochemistry and AFP levels were done once every 1 to 2 months. Contrast CT scan and chest X-ray were carried out once every 3 months for surveillance of tumor progression or metastasis. If residual viable tumor was detected on dynamic CT imaging, TACE was repeated in individuals who had compensated liver function.

\section{Statistical methods}

The $\mathrm{x}^{2}$ test or Fisher exact test (2-tailed) was used to compare categorical data, and the student's $t$ test was used for continuous data. Overall survival were calculated using the Kaplan-Meier method. Factors that appeared to be associated with survival on univariable analysis $(p<0.1)$ were introduced into the multivariable Cox proportional hazard model to determine the adjusted risk ratio. All statistical analyses were carried out using SPSS version 13.0 for Windows (SPSS Inc.; Chicago, IL). For all the tests, a $P$ value $<0.05$ was considered statistically significant.

\section{DISCUSSION}

Controversies exist in the management of patients with HCC with PVTT. Sorafenib is the only drug that improves survival in patients with advanced HCC for about 3 months. However, a SHARP trial demonstrated that tumor response to sorafenib treatment is only $2 \%$ $3 \%$ [11]. So consensus in China proposes comprehensive 
Table 1: Clinico-pathological features of the 10 patients who underwent liver resection

\begin{tabular}{cccccccccc}
\hline $\begin{array}{c}\text { Number of } \\
\text { patients }\end{array}$ & $\begin{array}{c}\text { Sex } \\
(\mathbf{F} / \mathbf{M})\end{array}$ & Year & HbsAg & $\begin{array}{c}\text { Tumor } \\
\text { number }\end{array}$ & $\begin{array}{c}\text { Tumor } \\
\text { Diameter }\end{array}$ & $\begin{array}{c}\text { PVTT } \\
\text { Type }\end{array}$ & $\begin{array}{c}\text { Surgical } \\
\text { procedure }\end{array}$ & $\begin{array}{c}\text { PVTT } \\
\text { Length }\end{array}$ & $\begin{array}{c}\text { Dying results } \\
\text { (Good/Mild/Poor) }\end{array}$ \\
\hline 1 & M & 45 & + & 1 & $5 \mathrm{~cm}$ & III & Thrombectomy & $3.2 \mathrm{~cm}$ & $\mathrm{G}$ \\
2 & M & 44 & + & 1 & $9 \mathrm{~cm}$ & II & En bloc & $2.2 \mathrm{~cm}$ & P \\
3 & M & 44 & + & 1 & $3.5 \mathrm{~cm}$ & II & En bloc & $2.7 \mathrm{~cm}$ & M \\
4 & M & 45 & + & 1 & $6 \mathrm{~cm}$ & II & En bloc & $2.6 \mathrm{~cm}$ & M \\
5 & M & 59 & + & 1 & $6 \mathrm{~cm}$ & II & En bloc & $1.6 \mathrm{~cm}$ & $\mathrm{G}$ \\
6 & M & 52 & + & 1 & $8 \mathrm{~cm}$ & II & En bloc & $2.4 \mathrm{~cm}$ & P \\
7 & M & 40 & + & 1 & $7 \mathrm{~cm}$ & III & Thrombectomy & $3.5 \mathrm{~cm}$ & M \\
8 & M & 65 & + & 2 & $6 \mathrm{~cm}$ & III & Thrombectomy & $2.8 \mathrm{~cm}$ & M \\
9 & M & 50 & + & 2 & $8 \mathrm{~cm}$ & III & Thrombectomy & $3.6 \mathrm{~cm}$ & $\mathrm{P}$ \\
10 & M & 48 & + & 3 & $8 \mathrm{~cm}$ & II & En bloc & $2.3 \mathrm{~cm}$ & $\mathrm{G}$ \\
\hline
\end{tabular}

Table 2: Clinico-pathological Features of the 2 subgroups who underwent TACE

\begin{tabular}{cccc}
\hline Variable & \multicolumn{3}{c}{ Lipiodol accumulation } \\
\cline { 2 - 4 } & Low $(\boldsymbol{n}=\mathbf{5 0})$ & High $(\boldsymbol{n}=\mathbf{2 7})$ & $\boldsymbol{P}$ Value \\
\hline Age(years) & $48.9 \pm 9.4$ & $51.4 \pm 9.6$ & 0.270 \\
Gender(n),Male:Female & $45 / 5$ & $24 / 3$ & 0.879 \\
AFP( $\mu \mathrm{g} / \mathrm{L}),<20: \geq 20$ & $8 / 42$ & $3 / 24$ & 0.440 \\
CEA( $\mu \mathrm{g} / \mathrm{L})$ & $4.4 \pm 13.8$ & $3.3 \pm 4.1$ & 0.705 \\
AST(IU/L) & $76.0 \pm 40.1$ & $85.5 \pm 47.3$ & 0.362 \\
ALT(IU/L) & $62.1 \pm 44.3$ & $59.1 \pm 30.3$ & 0.754 \\
Total bilirubin( $\mu$ mol/L) & $19.4 \pm 9.2$ & $19.4 \pm 9.4$ & 0.931 \\
HBsAg(n),Positive:Negative & $44 / 6$ & $23 / 4$ & 0.703 \\
Tumor number(n), Single:Multiple & $30 / 20$ & $14 / 13$ & 0.604 \\
Tumor diameter(cm) & $9.1 \pm 3.3$ & $9.0 \pm 3.5$ & 0.931 \\
TACE sessions, $1:>1$ & $25 / 25$ & $22 / 5$ & 0.014 \\
Classification of PVTT (n) & & & 0.171 \\
Type I & 9 & 11 & \\
Type II & 21 & 10 & \\
Type III & 14 & 6 & \\
Type IV & 6 & 0 & \\
\hline
\end{tabular}
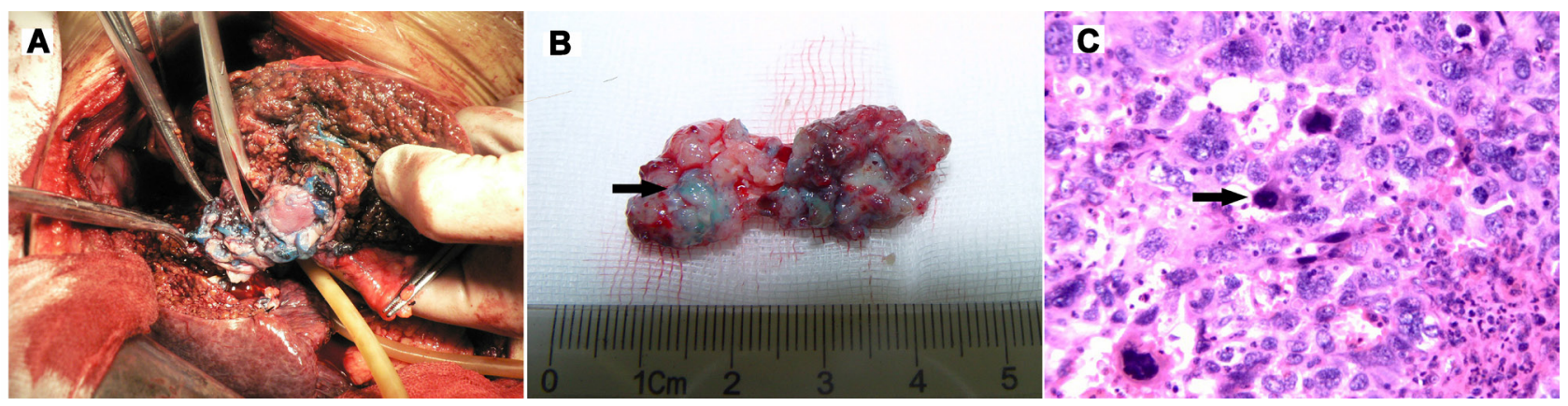

Figure 1: (A) Surgical procedure for removing PVTT. (B) More than 2/3 of The PVTT was dyed with methylene blue (Arrow). (C) Methylene blue was detected in microvessels microscopically (Arrow) 
Table 3: Survival of the 4 classes of lipiodol classification according to the 4 types of PVTT

\begin{tabular}{cccc}
\hline PVTT extent class & Lipiodol classification & N. patients & $\begin{array}{c}\text { Median Survival } \\
\text { (Month) }\end{array}$ \\
\hline \multirow{3}{*}{ Tpye I } & 1 & 2 & 13.5 \\
& 2 & 9 & 11.0 \\
& 3 & 6 & 5.8 \\
& 4 & 3 & 3.2 \\
\hline \multirow{2}{*}{ Tpye II } & 1 & 4 & 13.4 \\
& 2 & 6 & 6.4 \\
& 3 & 11 & 3.6 \\
\multirow{2}{*}{ Tpye III } & 4 & 10 & 2.7 \\
& 1 & 3 & 10.7 \\
& 2 & 3 & 6.6 \\
& 3 & 3 & 2.4 \\
& 4 & 11 & 4.8 \\
\hline \multirow{2}{*}{ Tpye IV } & 1 & 0 & NA \\
& 2 & 0 & NA \\
& 3 & 2 & 3.3 \\
& 4 & 4 & 2.2 \\
\hline
\end{tabular}

treatment of PVTT including TACE because evidence indicates that TACE can be performed safely and feasibly in select PVTT patients. The median OS obtained with sorafenib was 8.1 months in patients with PVTT in the SHARP, and the median OS was only 5.9 months in our study. We found that there are significant differences between different PVTT types, the median OS PVTT Type
I patients was 8.5 months and the Type IV was only 2.5 months. So we consider that there might be differences in PVTT types and tumor characters between these two studies.

The origin where the blood supply of PVTT comes from is unclear and possible sources include the hepatic artery, portal vein or other vessels around the porta hepatis.

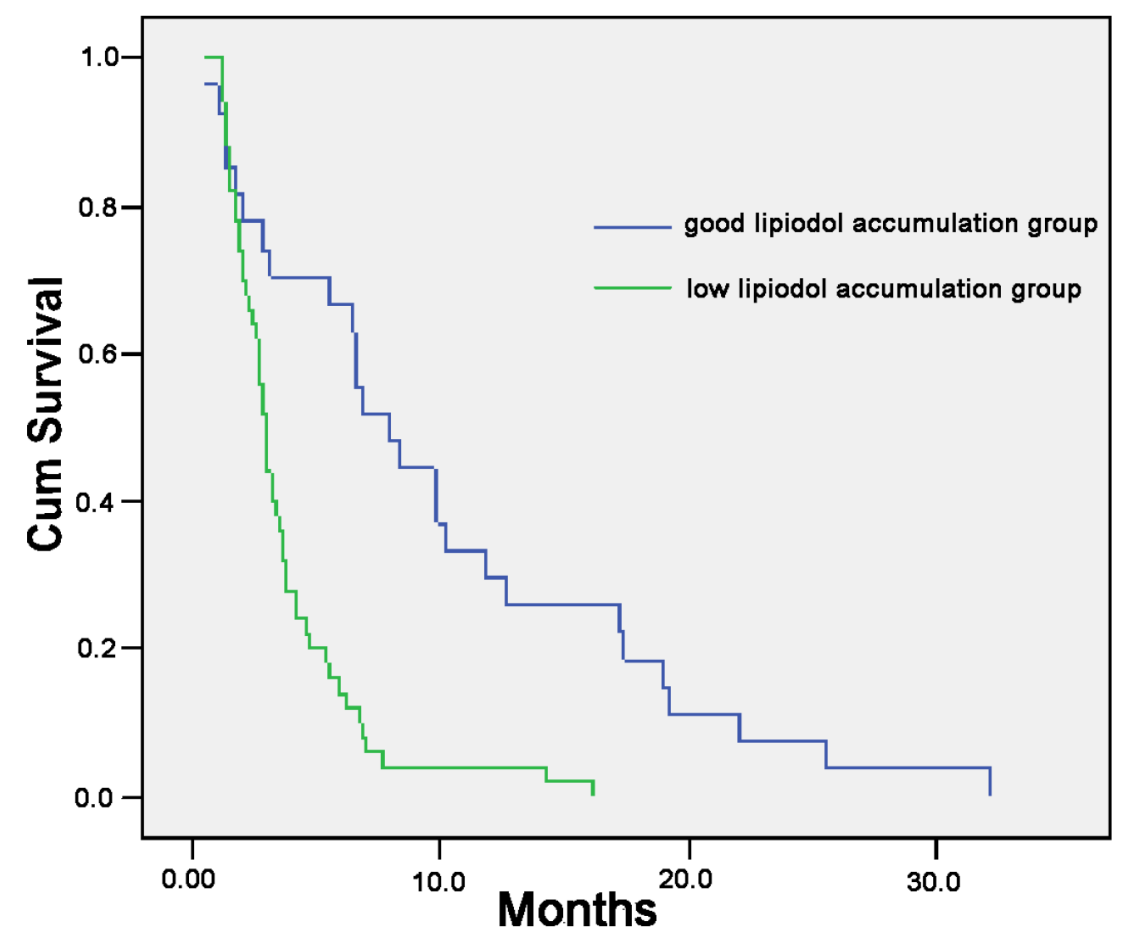

Figure 2: Overall survival according to different degrees of accumulation of Lipiodol in the PVTT 


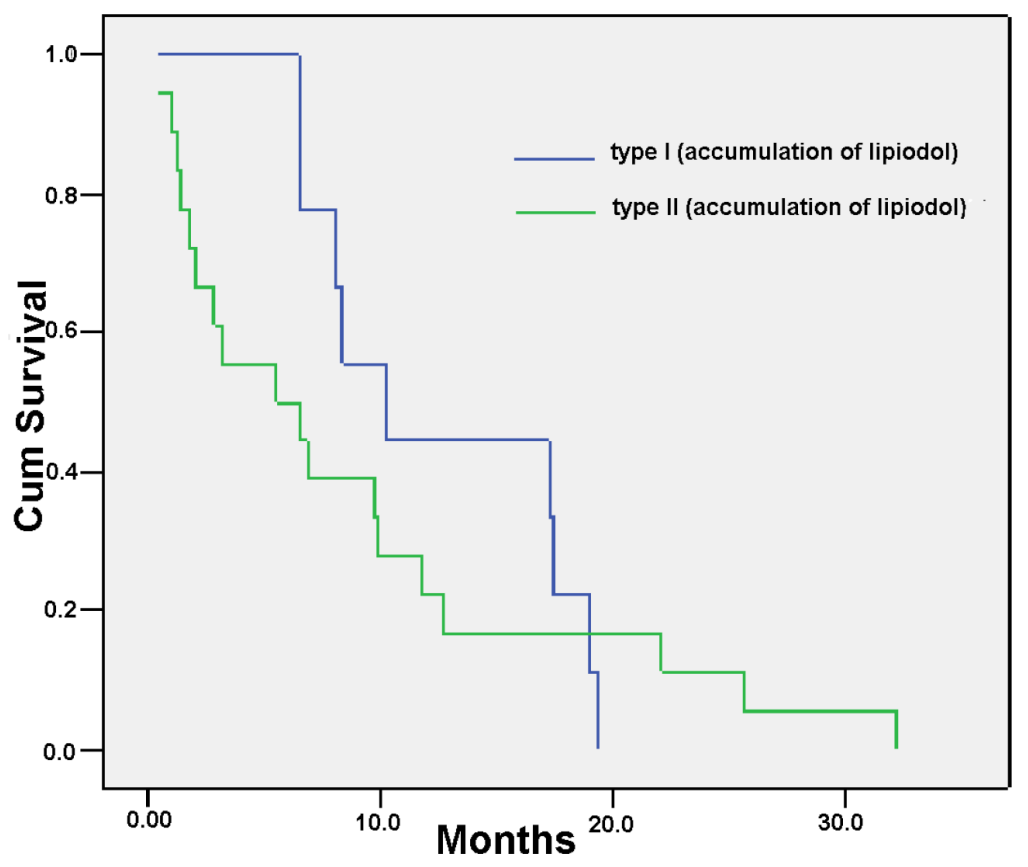

Figure 3: Overall survival according to different degrees of accumulation of Lipiodol in the PVTT

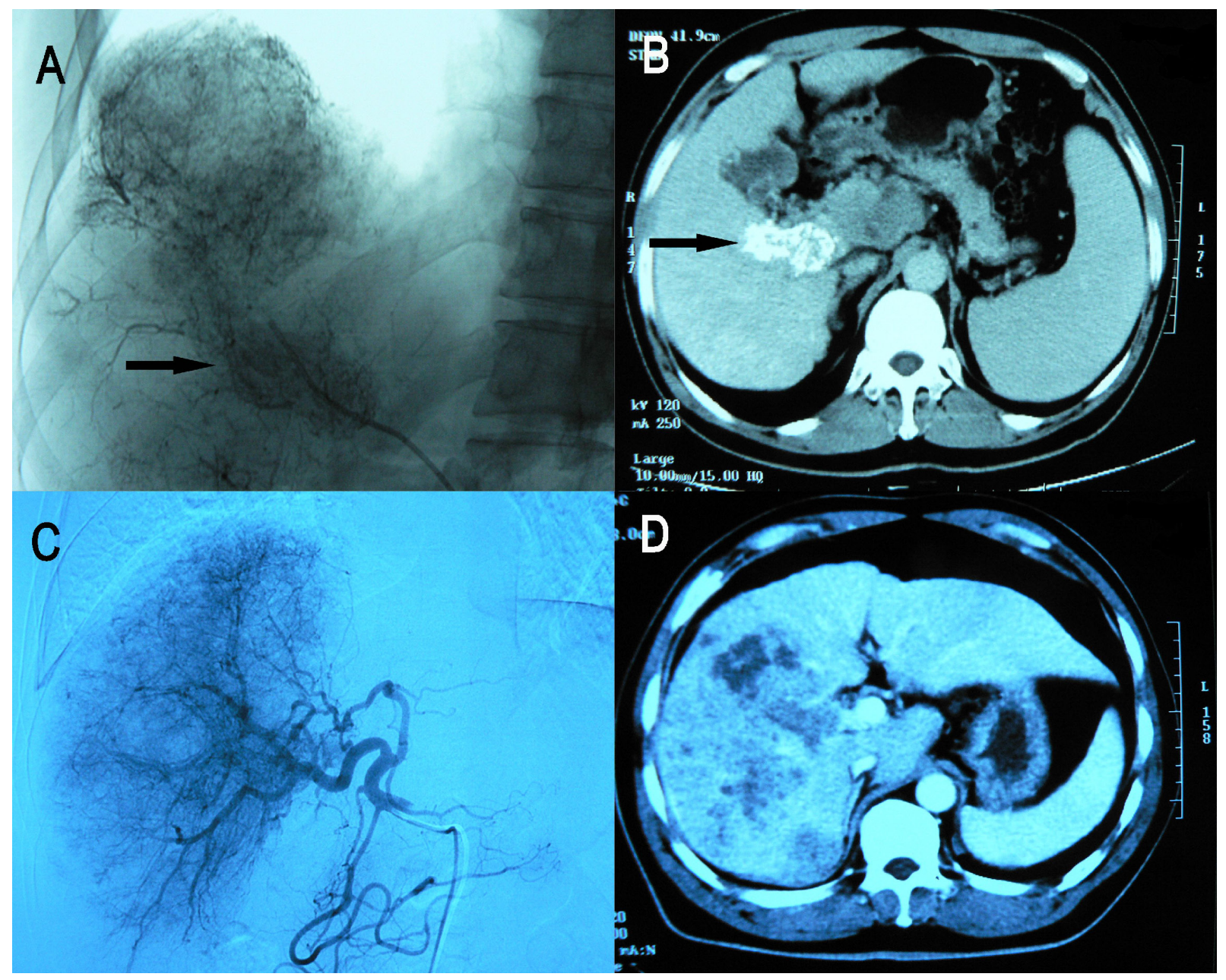

Figure 4: (A and B) PVTT was seen during digital subtraction angiography (DSA) and Lipiodol heavily accumulated in PVTT (OS, 17.4months); (C and D) PVTT was not seen during DSA and Lipiodol lightly accumulated in PVTT (OS, 1.5 month) 
Table 4: Univariate analysis of prognostic factors

\begin{tabular}{|c|c|c|c|c|c|}
\hline Variable & $n$ & $\begin{array}{l}\text { Median survival } \\
\text { (months) }\end{array}$ & HR & $95 \%$ CI & $\boldsymbol{P}$ \\
\hline Age, years, $<60: \geq 60$ & $47 / 30$ & $10.2 / 8.9$ & 0.996 & $0.969-1.024$ & 0.119 \\
\hline Gender,Male:Female & $69 / 8$ & $6.0 / 5.1$ & 0.952 & $0.442-2.383$ & 0.706 \\
\hline AFP, $\mu \mathrm{g} / \mathrm{L},<20: \geq 20$ & $12 / 65$ & $6.2 / 5.9$ & 1.000 & $1.000-1.001$ & 0.885 \\
\hline ALT, IU/L, $<44: \geq 44$ & $26 / 51$ & $5.3 / 6.0$ & 0.998 & $0.989-1.006$ & 0.368 \\
\hline AST, IU/L, <44: $\geq 44$ & $16 / 61$ & $4.18 / 6.4$ & 1.008 & $1.000-1.016$ & 0.063 \\
\hline Albumin, $\mathrm{g} / \mathrm{l},<35: \geq 35$ & $14 / 63$ & $4.26 / 6.31$ & 0.902 & $0.761-1.069$ & 0.236 \\
\hline Total bilirubin, $\mu \mathrm{mol} / \mathrm{L},<17.1: \geq 17.1$ & $38 / 39$ & $5.6 / 6.3$ & 1.009 & $0.983-1.037$ & 0.615 \\
\hline HBsAg,Positive : Negative & $67 / 10$ & $5.9 / 4.3$ & 0.829 & $0.376-1.827$ & 0.423 \\
\hline Tumor number, Single:Multiple & $43 / 34$ & $5.7 / 6.1$ & 0.817 & $0.491-1.359$ & 0.804 \\
\hline Diameter $(\mathrm{cm}), \leq 5:>5$ & $11 / 66$ & $10.2 / 5.4$ & 1.096 & $1.022-1.175$ & 0.031 \\
\hline TACE sessions $(n), 1:>1$ & $47 / 30$ & $6.6 / 4.8$ & 0.863 & $0.610-1.222$ & 0.226 \\
\hline Classification of PVTT $(n)$ & & & 1.241 & $0.927-1.661$ & 0.111 \\
\hline Type I & 20 & 8.5 & & & \\
\hline Type II & 31 & 5.1 & & & \\
\hline Type III & 20 & 5.6 & & & \\
\hline Type IV & 6 & 2.5 & & & \\
\hline Accumulation of Lipiodol $(n)$ & & & 1.733 & $1.347-2.231$ & 0.000 \\
\hline I & 9 & 12.5 & & & \\
\hline II & 18 & 8.7 & & & \\
\hline III & 22 & 4.0 & & & \\
\hline IV & 28 & 3.5 & & & \\
\hline
\end{tabular}

This study showed that in about one third of patients with HCC, the blood supply to PVTT comes mainly from the hepatic artery. In these patients, TACE is more efficacious in prolonging survival than those patients who had poor blood supply to PVTT from the hepatic artery. And in the surgical part, we validated that the flow of PVTT was in line with the lipiodol accumulation.

TACE is widely used in advanced hepatocellular carcinoma. In selected patients with good hepatic reserve and collateral circulation around the porta hepatis, it can be used in patients with HCC with PVTT. Georgiades [12] et al demonstrated that TACE was safe and effective for patients with $\mathrm{HCC}$ that had invaded the main portal vein. The 1 -month mortality was $0 \%$ and the median survival was 9.5 months. There was no TACE-related hepatic infarction or liver failure. Jia and his associates [13] showed that the degree of accumulation of Lipiodol in PVTT after TACE was an independent prognostic factor. Our study supported that a higher degree of Lipiodol accumulation in PVTT was associated with significantly better overall survival after TACE.

Evidence is now accumulating that the blood supply of PVTT is complicated. Imaging techniques like enhanced CT or ultrasound suggest that at least some blood supply comes from the hepatic artery. The exact percentage of the blood supply is unknown and it seems to vary amongst individuals. Our study showed that in about one third of patients with HCC with PVTT the blood supply comes mainly from the hepatic artery. For the other two thirds of patients, the blood supply probably comes mainly from the portal vein or from other vessels in the porta hepatis. Published data suggested that the tumor itself can form new vessels which come from differentiation of cancer stem cells [14]. Our study probably explains why only about one third of patients with PVTT responded well to TACE.

Many factors are related to Lipiodol accumulation including $[15,16,17]$ blood supply, structures of capillaries inside the tumor and clearance rate of Lipiodol by tumor cell et al. Generally, blood supply is considered to be one of the most important factors contributed to early Lipiodol accumulation. Also it was reported $\mathrm{d}^{15}$ that patients with good tumor vascularity are generally considered to have good lipiodol accumulation and therefore, a good prognosis. Our method of carrying out TACE is to use gelatin sponge particles and lipiodol as embolizing agents. Gelatin sponge particles produce temporary occlusion of tumor feeding vessels which lasts for about two weeks [18, 19]. Lipiodol, when injected into the hepatic artery, selectively remains more in tumor nodules for several weeks to over a year due to siphoning effect from hypervascularization of tumor 
vessels and absence of Kupffer cells inside tumor tissues $[20,21]$. This results in the embolic effects on small vessels. However, lipiodol, when injected into the hepatic artery of normal liver parenchyma, accumulates in the portal vessels by arterioportal communications and is gradually released into the systemic circulation via the hepatic sinusoids or undergoes phagocytosis by Kupffer cells. Thus, our study presently partly means that responding patients do better than non-responding patients which are mainly related to blood flow of the PVTT. The lipiodol is usually cleared within a week $[20,22,23]$. Thus, the way that we carried out preoperative TACE in the 10 patients with resectable HCC with PVTT should not affect the subsequent study using intrahepatic arterial injection of methylene blue to study the arterial blood supply of PVTT in the resected specimens.

This paper has limitations. Firstly, we only consider only from the aspect of blood supply in the study and we will do further research in the future ;secondly, the sample size is small and in the majority of our patients, the HCC was hepatitis B related; lastly, the accuracy in measuring the dyed area is not very high. . Whether our results can be used in HCC due to other etiologies is unknown.

\section{CONCLUSIONS}

In conclusion, in about one third of patients, the blood supply of PVTT came from the hepatic artery. These patients responded better to TACE.

\section{ETHICAL APPROVAL}

Not needed.

\section{CONFLICTS OF INTEREST}

No benefits in any form have been received or will be received from a commercial party related directly or indirectly to the subject of this article.

\section{FUNDING}

This work is supported by the grants of The National Key Basic Research Program "973 project”(No: 2015CB554000), The Science Fund for Creative Research Groups (No: 81521091), Shanghai Science and Technology Committee ( No.134119a0200), and supported by shanghai shenkang group project (SHDC12015106). Collaborative Innovation Center for Cancer Medicine.

\section{REFERENCES}

1. Torre LA, Bray F, Siegel RL, Ferlay J, Lortet-Tieulent J, Jemal A. Global cancer statistics, 2012. CA Cancer J Clin. 2015; 65:87-108.

2. Zhang ZM, Lai EC, Zhang C, Yu HW, Liu Z, Wan BJ, Liu LM, Tian ZH, Deng H, Sun QH, Chen XP. The strategies for treating primary hepatocellular carcinoma with portal vein tumor thrombus. Int J Surg. 2015; 20:8-16.

3. Sun JX, Shi J, Li N, Guo WX, Wu MC, Lau WY, Cheng SQ. Portal vein tumor thrombus is a bottleneck in the treatment of hepatocellular carcinoma. Cancer Biol Med. 2016; 13:452-458.

4. Cheng S, Chen M, Cai J, National Research Cooperative Group for Diagnosis and Treatment of Hepatocellular Carcinoma with Tumor Thrombus. Chinese expert consensus on multidisciplinary diagnosis and treatment of hepatocellular carcinoma with portal vein tumor thrombus: 2016 edition. Oncotarget. 2017; 8:8867-8876. https://doi. org/10.18632/oncotarget.12817.

5. Bruix J, Sherman M, American Association for the Study of Liver Diseases. Management of hepatocellular carcinoma: an update. Hepatology. 2011; 53:1020-22.

6. Chung GE, Lee JH, Kim HY, Hwang SY, Kim JS, Chung JW, Yoon JH, Lee HS, Kim YJ. Transarterial chemoembolization can be safely performed in patients with hepatocellular carcinoma invading the main portal vein and may improve the overall survival. Radiology. 2011; 258:627-34.

7. Luo J, Guo RP, Lai EC, Zhang YJ, Lau WY, Chen MS, Shi M. Transarterial chemoembolization for unresectable hepatocellular carcinoma with portal vein tumor thrombosis: a prospective comparative study. Ann Surg Oncol. 2011; 18:413-20.

8. Chern MC, Chuang VP, Liang CT, Lin ZH, Kuo TM. Transcatheter arterial chemoembolization for advanced hepatocellular carcinoma with portal vein invasion: safety, efficacy, and prognostic factors. J Vasc Interv Radiol. 2014; 25:32-40.

9. Garin E, Rolland Y, Edeline J, Icard N, Lenoir L, Laffont S, Mesbah H, Breton M, Sulpice L, Boudjema K, Rohou T, Raoul JL, Clement B, Boucher E. Personalized dosimetry with intensification using 90Y-loaded glass microsphere radioembolization induces prolonged overall survival in hepatocellular carcinoma patients with portal vein thrombosis. J Nucl Med. 2015; 56:339-46.

10. Nishimine $K$, Uchida $H$, Matsuo $N$, Sakaguchi $H$, Hirohashi S, Nishimura Y, Guo Q, Ohishi H, Nagano N, Yoshioka T, Ohue S, Fukui H, Tsujii T. Segmental transarterial chemoembolization with Lipiodol mixed with anticancer drugs for nonresectable hepatocellular carcinoma: follow-up CT and therapeutic results. Cancer Chemother Pharmacol. 1994; 33:S60-68.

11. Llovet JM, Ricci S, Mazzaferro V, Hilgard P, Gane E, Blanc JF, de Oliveira AC, Santoro A, Raoul JL, Forner A, Schwartz M, Porta C, Zeuzem S, et al. Sorafenib in advanced hepatocellular carcinoma. N Engl J Med. 2008; 359:378-90.

12. Georgiades CS, Hong K, D'Angelo M, Geschwind JF. Safety and efficacy of transarterial chemoembolization in patients with unresectable hepatocellular carcinoma and portal vein thrombosis. J Vasc Interv Radiol. 2005; 16:1653-9. 
13. Jia L, Kiryu S, Watadani $\mathrm{T}$, Akai H, Yamashita $\mathrm{H}$, Akahane M, Ohtomo K. Prognosis of hepatocellular carcinoma with portal vein tumor thrombus:assessment based on clinical and computer tomography characteristics. Acta Med Okayama. 2012; 66:131-41.

14. Wang $\mathrm{R}$, Chadalavada $\mathrm{K}$, Wilshire J, Kowalik U, Hovinga KE, Geber A, Fligelman B, Leversha M, Brennan C, Tabar V. Glioblastoma stem-like cells give rise to tumour endothelium. Nature. 2010; 468:829-33.

15. Chen CS, Li FK, Guo CY, Xiao JC, Hu HT, Cheng HT, Zheng L, Zong DW, Ma JL, Jiang L, Li HL. Tumor vascularity and lipiodol deposition as early radiological markers for predicting risk of disease progression in patients with unresectable hepatocellular carcinoma after transarterial chemoembolization. Oncotarget. 2016; 7:7241-52. https:// doi.org/10.18632/oncotarget.6892.

16. Kim HC, Chung JW, Lee W, Jae HJ, Park JH. Recognizing extrahepatic collateral vessels that supply hepatocellular carcinoma to avoid complications of transcatheter arterial chemoembolization. Radiographics. 2005; 25:S25-39.

17. Hu HT, Kim JH, Lee LS, Kim KA, Ko GY, Yoon HK, Sung KB, Gwon DI, Shin JH, Song HY. Chemoembolization for hepatocellular carcinoma: multivariate analysis of predicting factors for tumor response and survival in a 362-patient cohort. J Vasc Interv Radiol. 2011; 22:917-923.
18. Tsochatzis E, Meyer, T, Marelli L, Burroughs AK. Which transarterial therapy is best for hepatocellular carcinoma? the evidence to date. J Hepatol. 2010; 53:588.

19. Coldwell DM, Stokes KR, Yakes WF. Embolotherapy: agents, clinical applications, and technique. Radiographics. 1994; 14:623-43.

20. Kan Z, McCuskey PA, Wright KC, Wallace S. Role of Kupffer cells in iodized oil embolization. Invest Radiol. 1994; 29:990-3.

21. Ohishi H, Uchida H, Yoshimura H, Ohue S, Ueda J, Katsuragi M, Matsuo N, Hosogi Y. Hepatocellular carcinoma detected by iodized oil. Use of anticancer agents. Radiology. 1985; 154:25-9.

22. Shin SW. The current practice of transarterial chemoembolization for the treatment of hepatocellular carcinoma. Korean J Radiol. 2009; 10:425-34.

23. Okayasu I, Hatakeyama S, Yoshida T, Yoshimatsu S, Tsuruta K, Miyamoto H, Kimula Y. Selective and persistent deposition and gradual drainage of iodized oil, Lipiodol in the hepatocellular carcinoma after injection into the feeding hepatic artery. Am J Clin Pathol. 1988; 90:536-44. 